\title{
Prevalência de SOBRePESO E OBESIDADE NAS RegIÕES NORDESTE E SUdESTE dO BRASIL
}

\author{
Marcelo Militão Abrantes, Joel Alves lamounier*, Enrico Antônio Colosimo \\ Trabalho realizado na Faculdade de Medicina da Universidade Federal de Minas Gerais, Belo Horizonte, MG.
}

RESUMO - OBjetivo. Avaliar a prevalência de sobrepeso e obesidade em crianças, adolescentes, adultos e idosos brasileiros das regióes Nordeste e Sudeste;

Métodos. Avaliação antropométrica de 17.184 pessoas, estudadas na Pesquisa sobre Padrões de Vida (PPV) realizada pelo IBGE em 1996/97, através do índice de massa corporal sendo usados como limite para sobrepeso e obesidade os valores propostos por Cole et al. por permitirem uma continuidade de critério de sobrepeso e obesidade na infância, adolescência e idade adulta.

Resultados. Predomínio do sexo feminino, 53,1\%, na população estudada. A média de idade foi de 29 anos e 5 meses (desvio padrão de \pm 20 anos), a mediana foi de 25 anos e 6 meses. A prevalência de sobrepeso foi de $10,8 \%$ entre crianças, $9,9 \%$ nos adolescentes e
$28,3 \%$ entre adultos e a de obesidade foi $7,3 \%, 1,8 \%$ e $9,7 \%$, respectivamente. Observado um aumento gradativo da prevalência de sobrepeso e obesidade desde a infância até a idade adulta, com declínio entre os idosos.

Conclusöes. Prevalência conjunta de sobrepeso e obesidade na população brasileira é maior no sexo feminino sendo que mais da metade das mulheres das regiōes Nordeste e Sudeste do Brasil, com idade entre 50 e 69 anos, têm sobrepeso e/ou obesidade. A comparação de estudos de prevalência de sobrepeso e obesidade na infância e adolescência é dificultada pela escassez de estudos populacionais nacionais e diversidade de critérios utilizados na avaliação nutricional.

UnITERMOS: Obesidade. Sobrepeso. Avaliação nutricional.

\section{INTRODUÇÃO}

Aobesidade é considerada um importante problema de saúde pública em países desenvolvidos e uma epidemia global pela Organização Mundial de Saúde (OMS)'. O aumento de sua prevalência em países em desenvolvimento, especialmente na América Latina, também já foi estudado, e em países como Índia e China o aumento de $1 \%$ na prevalência de obesidade gera 20 milhões de novos casos ${ }^{2,3,4}$.

Está associada com hipertensão arterial, doença cardíaca, oesteoartrite, diabetes tipo 2 e alguns tipos de câncer ${ }^{1,5}$. Pessoas obesas, particularmente crianças e adolescentes, freqüentemente apresentam baixa auto-esti$\mathrm{ma}$, afetando a performance escolar e relacionamentos levando a conseqüências psicológicas a longo prazo. Em algumas comunidades pessoas obesas podem também sofrer discriminação social 6 .

AOMS indica a antropometria como método mais útil para identificar pessoas obesas pois é o mais barato, não-invasivo, universal-

*Correspondência:

Departamento de Pediatria - Faculdade de Medicina da UFMG

Av. Alfredo Balena 190, Belo Horizonte MG - Brazil CEP 30130-100 mente aplicável e com boa aceitação pela população. Índices antropométricos são obtidos a partir da combinação de duas ou mais informações antropométricas básicas (peso, sexo, idade, altura)'.

Atualmente, o índice mais utilizado para identificar pessoas obesas é o índice de massa corporal (IMC), calculado pela fórmula peso (em kg) dividido pelo quadrado da altura (em metros), desenvolvido no século passado, por Lambert Adolphe Jacques Quetelet, matemático belga ${ }^{7}$. Este índice tem seu uso praticamente consensual na avaliação nutricional de adultos cujos limites inferior e superior da normalidade são baseados em critérios estatísticos que correlacionam uma maior morbimortalidade em pessoas com IMC acima ou abaixo deste intervalo $0^{1,8-11}$. Seu uso em adolescentes e crianças começou a ser mais difundido após a publicação de Must et a $\left.\right|^{\mid 2,13}$, que apresentaram valores de percentis por idade e sexo. Recentemente, para definir obesidade na infância e adolescência, diversos autores manifestam uma tendência em utilizar critérios estatísticos de mortalidade e de continuidade dos valores de IMC com os adotados na idade adulta ${ }^{14-20}$. Cole et al (2000) propuseram valores de ponto de corte concordantes com esta tendência ${ }^{20}$.
O objetivo do presente estudo é avaliar a prevalência de sobrepeso e obesidade em crianças, adolescentes, adultos e idosos brasileiros das regiões Nordeste e Sudeste.

\section{MÉtodos}

Para o presente estudo foram utilizados dados da Pesquisa sobre Padrões de Vida (PPV), realizada pelo IBGE em convênio com - Banco Mundial. Esta pesquisa avaliou as condições de moradia, tendências demográficas (migração, fecundidade e história de nascimentos), acesso aos serviços de educação e saúde, nutrição, condições de vida da população brasileira, além de dados de peso e altura ${ }^{21}$.

A amostra da pesquisa foi constituída por 19.409 pessoas residentes em 5.000 domicílios, distribuídos em 554 setores censitários das regiões Nordeste e Sudeste do País. A amostra foi selecionada de maneira randomizada e sua metodologia está descrita em outra publicação ${ }^{21}$. Os dados desta pesquisa foram disponibilizados via CD-ROM pelo IBGE.

Os valores referentes ao peso, altura e sexo foram extraídos do banco de dados principal e armazenados em um banco de dados secundário no software Epilnfo, versão 
ObesidAde E SOBREPESO No BRASIL

\begin{tabular}{|c|c|c|c|c|}
\hline \multirow[t]{2}{*}{ Idade (anos) } & \multicolumn{2}{|c|}{ Sobrepeso } & \multicolumn{2}{|c|}{ Obesidade } \\
\hline & Masculino & Feminino & Masculino & Feminino \\
\hline 2 & $|8,4|$ & 18,02 & 20,09 & $|9,8|$ \\
\hline 2,5 & 18,13 & 17,76 & 19,80 & 19,55 \\
\hline 3 & 17,89 & 17,56 & 19,57 & 19,36 \\
\hline 3,5 & 17,69 & 17,40 & 19,39 & 19,23 \\
\hline 4 & 17,55 & 17,28 & 19,29 & 19,15 \\
\hline 4,5 & 17,47 & 17,19 & 19,26 & 19,12 \\
\hline 5 & 17,42 & 17,15 & 19,30 & 19,17 \\
\hline 5,5 & 17,45 & 17,20 & 19,47 & 19,34 \\
\hline 6 & 17,55 & 17,34 & 19,78 & 19,65 \\
\hline 6,5 & $|7,7|$ & 17,53 & 20,23 & 20,08 \\
\hline 7 & 17,92 & 17,75 & 20,63 & 20,51 \\
\hline 7,5 & 18,16 & 18,03 & 21,09 & 21,01 \\
\hline 8 & 18,44 & 18,35 & 21,60 & 21,57 \\
\hline 8,5 & 18,76 & 18,69 & 22,17 & 22,18 \\
\hline 9 & 19,10 & 19,07 & 22,77 & 22,81 \\
\hline 9,5 & 19,46 & 19,45 & 23,39 & 23,46 \\
\hline 10 & 19,84 & 19,86 & 24,00 & $24, I I$ \\
\hline 10,5 & 20,20 & 20,29 & 24,57 & 24,77 \\
\hline 11 & 20,55 & 20,74 & 25,10 & 25,42 \\
\hline 11,5 & 20,89 & 21,20 & 25,58 & 26,05 \\
\hline 12 & 21,22 & 21,68 & 26,02 & 26,67 \\
\hline 12,5 & 21,56 & 22,14 & 26,02 & 26,67 \\
\hline 13 & 21,91 & 22,58 & 26,84 & 27,76 \\
\hline 13,5 & 22,27 & 22,98 & 27,25 & 28,20 \\
\hline 14 & 22,62 & 23,34 & 27,63 & 28,57 \\
\hline $\mid 4,5$ & 22,96 & 23,66 & 27,98 & 28,87 \\
\hline 15 & 23,29 & 23,94 & 28,30 & 29,11 \\
\hline 15,5 & 23,60 & 24,17 & 28,60 & 29,29 \\
\hline 16 & 23,90 & 24,37 & 28,88 & 29,43 \\
\hline 16,5 & 24,19 & 24,54 & 29,14 & 29,56 \\
\hline 17 & 24,46 & 24,70 & 29,41 & 29,69 \\
\hline 17,5 & 24,73 & 24,85 & 29,70 & 29,84 \\
\hline $18 e>18$ & 25,00 & 25,00 & 30,00 & 30,00 \\
\hline
\end{tabular}

Fonte:Coleetal.(2000)

6.0422. O IMC foi calculado pela fórmula: peso/(altura) ${ }^{2}$. A variável idade, inicialmente calculada em meses, foi obtida pela diferença entre a data do exame e data de nascimento.

Para classificar o estado nutricional foi desenvolvido um programa no software Epilnfo sendo utilizados os valores propostos por Cole et al., que permitem uma continuidade de critério de sobrepeso e obesidade na infância, adolescência e idade adulta $^{20}$. Estes valores de IMC estão apresentados na Tabela I.

Considerado eutrófico o IMC abaixo dos valores propostos como limite para sobre- peso, como sobrepeso o IMC igual ou acima dos valores do limite para sobrepeso e abaixo do limite para obesidade e como obeso o IMC igual ou acima dos valores propostos como limite para obesidade.

Não foram analisados dados de 634 crianças com idade inferior a dois anos, pois Cole et al. propuseram valores para crianças com dois anos ou mais. Outras I.59I pessoas não foram consideradas na análise porque não tinham informações sobre peso e/ou altura. Sendo assim, a amostra final do presente estudo foi de 17.184 pessoas.

\section{Resultados}

Do total de 17.184 pessoas avaliadas, 2.683 (I5,7\%) eram crianças (idade menor que 10 anos), 3.943 (23\%) adolescentes (idade entre 10 anos e menor que 20 anos) e 10.522 (61,3\%) adultos (acima de 20 anos).

A distribuição entre os dois sexos foi: $8.063(46,9 \%)$ do sexo masculino e 9.121 $(53,1 \%)$ do sexo feminino. A média de idade foi de 29 anos e 5 meses (desvio padrão de \pm 20 anos), a mediana foi de 25 anos e 6 meses. A prevalência de crianças, adolescentes e adultos com sobrepeso e obesos, agrupadas por idade e sexo estão nas Tabelas 2, 3 e 4. 
Tabela 2 - Prevalência de crianças com sobrepeso e obesidade nas regióes Nordeste e Sudeste do Brasil, segundo valores propostos por Cole et al. (2000)

\begin{tabular}{cccccc}
\hline Idade(anos) & \multicolumn{2}{c}{ Masculino } & & \multicolumn{2}{c}{ Feminino } \\
\cline { 2 - 3 } \cline { 5 - 6 } 2 & Sobrepeso & Obesidade & & Sobrepeso & Obesidade \\
3 & 16,6 & 11,2 & & 17,4 & 9,0 \\
4 & 13,3 & 5,3 & 7,4 & 6,4 \\
5 & 10,6 & 6,5 & 11,6 & 11,6 \\
6 & 7,0 & 8,6 & 12,3 & 7,3 \\
7 & 6,5 & 12,4 & 7,4 & 11,1 \\
8 & 8,8 & 9,9 & & 11,4 & 7,8 \\
9 & 9,8 & 4,7 & 7,2 & 7,8 \\
Total & 12,6 & 2,6 & 12,2 & 2,8 \\
\hline
\end{tabular}

Tabela 3 - Prevalência de adolescentes com sobrepeso e obesidade nas regiões Nordeste e Sudeste do Brasil, segundo valores propostos por Cole et al. (2000)

\begin{tabular}{cccccc}
\hline Idade(anos) & \multicolumn{2}{c}{ Masculino } & & \multicolumn{2}{c}{ Feminino } \\
\cline { 2 - 4 } \cline { 5 - 6 } & Sobrepeso & Obesidade & & Sobrepeso & Obesidade \\
10 & 11,6 & 2,6 & & 11,9 & 5,1 \\
11 & 8,9 & 2,7 & & 11,4 & 2,2 \\
12 & 8,9 & 2,5 & & 10,7 & 1,7 \\
13 & 7,0 & 1,5 & & 12,3 & 2,6 \\
14 & 9,3 & 0,5 & 14,4 & 2,4 \\
15 & 7,5 & 1,3 & & 9,9 & 1,3 \\
16 & 11,2 & 2,0 & 7,9 & 0,5 \\
17 & 6,8 & 1,7 & 7,5 & 2,6 \\
18 & 8,3 & 0,6 & 9,9 & 1,7 \\
19 & 7,7 & 1,7 & 16,1 & 2,1 \\
Total & 8,6 & 1,6 & 11,3 & 1,9 \\
\hline
\end{tabular}

Tabela 4 - Prevalência de adultos com sobrepeso e obesidade nas regióes Nordeste e Sudeste do Brasil.

\begin{tabular}{cccccc}
\hline Idade(anos) & \multicolumn{2}{c}{ Masculino } & & \multicolumn{2}{c}{ Feminino } \\
\cline { 2 - 3 } $20-29$ & Sobrepeso & Obesidade & & Sobrepeso & Obesidade \\
$30-39$ & 22,3 & 3,4 & 17,3 & 6,6 \\
$40-49$ & 32,2 & 7,8 & 24,8 & 10,7 \\
$50-59$ & 33,8 & 9,4 & 30,7 & 17,2 \\
$60-69$ & 35,5 & 8,9 & 35,3 & 18,6 \\
$70-79$ & 35,8 & 6,4 & 35,9 & 19,2 \\
$80-$ & 29,6 & 5,4 & 27,5 & 14,7 \\
Total & 16,5 & 0,9 & 27,2 & 5,7 \\
\hline
\end{tabular}

Observou-se um aumento gradativo da prevalência de sobrepeso e obesidade desdea infância até a idade adulta, com declínio entre os idosos (Figura I).

\section{Discussão}

A OMS defende que a definição de obesidade em crianças e adolescentes tenha uma relação com a definição em adultos: "como as doenças no período da adolescência são escassas, é particularmente importante o quanto a antropometria na adolescência pode predizer fatores de risco ou doenças na idade adulta", citando ainda que "pouco é conhecido sobre níveis específicos de IMC na adolescência e sua relação com risco futuro ou concomitante e resposta a intervenções"'. Diversos autores também têm manifestado preferência por tentar definir o ponto de corte baseado em critérios estatísticos de mortalidade e de continuidade com a idade adulta $9,10,11,20$. Cole et al. foram um dos primeiros autores a apresentar valores de IMC concordantes com esta tendência ${ }^{20}$. No entanto, ainda não está determinado se a continuidade de critério entre infância e idade adulta é acompanhada pelo mesmo risco de morbi-mortalidade ${ }^{23}$.

Apesar desta tendência, existem outras publicações com valores limítrofes de IMC que também são utilizados para identificar criançase adolescentes com sobrepeso e obesidade ${ }^{\text {14-19 }}$. Por isso alguns autores consideram que uma estimativa internacional da prevalência de obesidade pediátrica e comparação de prevalência e tendência secular de obesidade pediátrica não são possíveis devido a diversidade de critérios usados ${ }^{24,25}$. No presente estudo utilizou-se os valores propostos por Cole et al. porque eles permitem uma continuidade de definição de obesidade na infância, adolescência ${ }^{20}$.

Dados brasileiros com relação à obesidade na infância e adolescência são ainda limitados e a maioria dos estudos nacionais são baseados em amostras de estudantes, ealguns estudaram somente as escolas privadas. Além disso a diversidade de critérios utilizados na definição de obesidade infantil acarreta dificuldades na comparação dos resultados do presente estudo com publicações nacionais e internacionais ${ }^{26}$.

No presente estudo, a prevalência de sobrepeso e obesidade entre 2 e 10 anos de idade foi semelhante nos dois sexos. Nos adolescentes, a prevalência de sobrepeso e obesi- 
Figura I - Prevalência de sobrepeso e obesidade em brasileiros das regiöes Nordeste e Sudeste, agrupados por sexo e faixa etária-1997

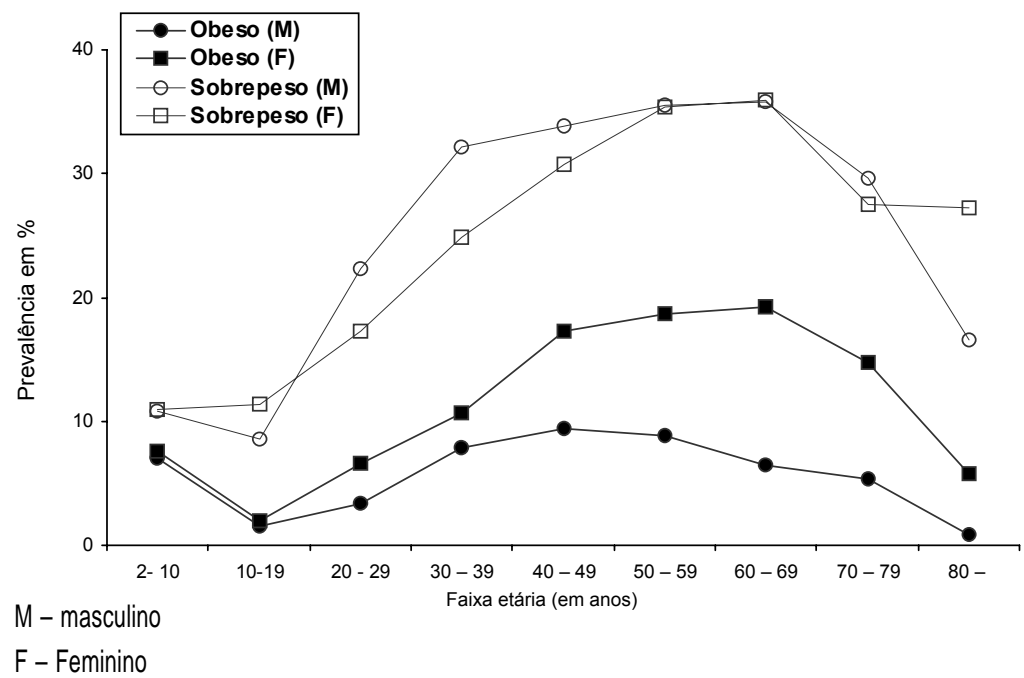

dade foi maior no sexo feminino, exceto aos 16 anos de idade, o que já foi descrito por alguns autores ${ }^{6,27}$. Outros autores descrevem 0 oposto: prevalência maior de sobrepeso em adolescentes do sexo masculino ${ }^{28}$. Alguns autores citam prevalência de obesidade superior (I4\% paraadolescentes masculinos entre $17 \mathrm{e}$ 19 anos) à encontrada no presente estudo 29.

Alguns autores descrevem que a prevalência de sobrepeso começa cedo, por volta dos 5-6 anos de idade, especialmente em meninas 6 , o que não foi encontrado no presente estudo; pelo contrário, nesta faixa etária foi encontrada a menor prevalência de sobrepeso em toda a infância. Na China, a maior prevalência de sobrepeso e obesidade entre adolescentes é relatada entre II e 12 anos $^{30}$ que também não foi observada neste estudo.

Usando outros valores de $\mathrm{IMC}^{12,1}$ em crianças de 6 a 19 anos de uma escola particular em Recife, encontrou-se uma prevalência de $26,2 \%$ de sobrepeso e $8,5 \%$ de obesidade, sendo maior em crianças do que nos adolescentes: $34,3 \%$ contra $14,2 \%$ e $20 \%$ contra $4,2 \%$, respectivamente ${ }^{2}$ ). No presente estudo a prevalência de obesidade foi maior em crianças do que em adolescentes enquanto a prevalência de sobrepeso foi praticamente igual nas duas faixas etárias. (Tabelas I e 2). A prevalência de sobrepeso e obesidade em estudos populacionais baseados em valores de IMC propostos por Cole et al ${ }^{20}$ e Must, Dallal e Dietz ${ }^{12,13}$ podem ser comparadas ${ }^{31}$

Ao contrário do que ocorre na infância e adolescência, a comparação de estudos de prevalência de sobrepeso e obesidade em adultos e idosos é facilitada pela uniformidade de critérios (valores de IMC).

No presente estudo a prevalência de obesidade foi maior em mulheres do que em homens adultos (>20 anos). Resultados semelhantes são descritos por outros autores $^{6,27,32-36}$. Um estudo nacional, envolvendo 224 indígenas de duas comunidades $X$ avante no Estado de Mato Grosso, mostrou distribuição igualitária de sobrepeso e obesidade entre homens e mulheres em uma comunidade e maior no sexo feminino, em outra ${ }^{37}$. Em bancários, a prevalência de sobrepeso e obesidade (IMC > 25) é maior em pessoas do sexo masculino do que feminino, atingindo $68,7 \%$ em homens com 40 anos ou mais ${ }^{38}$. Quando se estuda a estratificação por renda, verifica-se um incremento da prevalência de sobrepeso/obesidade com o aumento de renda para homens, mas não para mulheres $^{36,38,39}$. Na Arábia Saudita, foi descrita a influência do estado marital: pessoas casadas apresentaram prevalência de sobrepeso e obesidade maior que pessoas solteiras (35,4\% x $19,3 \%$ e $25,1 \%$ × $8,9 \%$ para muIheres e homens, respectivamente) ${ }^{6}$.

Considerando sobrepeso e obesidade conjuntamente (IMC >25), mais da metade das mulheres da Tunísia e Marrocos estão neste intervalo ${ }^{27}$. Isto também foi encontrado no presente estudo na faixa etária entre $50 \mathrm{e}$ 79 anos, de idade (Tabela 3). Esta elevada prevalência de sobrepeso e obesidade (mais da metade), também foi descrita em indígenas brasileiros do sexo masculino ${ }^{37}$.

No presente estudo a prevalência de obesidade mantém-se praticamente estável entre os homens com idade entre 30 e 59 anos, diminuindo a partir desta idade, enquanto nas mulheres ocorre aumento progressivo da prevalência de obesidade até a idade de 70 anos quando começa a declinar. Achado semelhante foi descrito na Arábia Saudita 6 . Esta diferença na prevalência de obesidade entre o sexo masculino e feminino também foi descrita em outro estudo nacional, mas com faixa etária um pouco diferente: no sexo masculino a prevalência estabilizou por volta dos 40 anos de idade enquanto no sexo feminino a prevalência dobrou a partir desta idade ${ }^{36}$. Na Arábia Saudita, a maior prevalência de obesidade (33,7\%) foi descrita na quinta década de vida e a menor entre 18 e 29 anos de idade $(10,6 \%)^{6}$.

A prevalência de obesidade em adultos varia entre $10 \%$ e $25 \%$ na maioria dos países do Oeste Europeu e entre $20 \%$ e $25 \%$ em alguns países daAmérica 6 . No presente estudo a prevalência de obesidade em adultos $(>20$ anos) foi de $6,7 \%$ e de sobrepeso foi de $30 \%$.

Oaumento da prevalência de sobrepesoe obesidade na idade adulta e sua diminuição entre os idosos também já foi descrito por outros autores ${ }^{1,32,36}$. (Figura. I)

\section{Conclusóes}

Conclui-se que a prevalência conjunta de sobrepeso e obesidade na população brasileira é maior no sexo feminino, atingindo índices preocupantes, uma vez que mais da metade das mulheres das regiões Nordeste e Sudeste do Brasil, com idade entre 40 e 79 anos, têm sobrepeso (IMC $>25)$.

A prevalência de obesidade no sexo masculino estabiliza-se mais precocemente que no sexofeminino.

A comparação de estudos de prevalência de sobrepeso e obesidade na infância e adolescência é dificultada pela escassez de estudos populacionais nacionais e diversidade de critérios utilizados na avaliação nutricional.

Os valores propostos por Cole et al permitem uma continuidade na definição de 
Abrantes MMet al.

sobrepeso e obesidade na infância, adolescência e idade adulta, mas ainda não está determinado se esta continuidade é acompanhada pelo risco de morbi-mortalidade ${ }^{23}$. Outros estudos ainda são necessários para se saber qual o melhor valor para definir sobrepeso e obesidade na infância e adolescência.

\section{SUMMARY}

OVerWeight AND OBESTTY PREVAlenCE IN Northeast and Southeast Regions of Brazl

OBJECTIVE. to evaluate the prevalence of overweight and obesity in children, adolescents, adults and elderlies from Northeast and Southeastregions of Brazil.

Methods: anthropometric evaluation of 17. I84 people, studied in Life Pattern Research conducted by Brazilian Institute of Geographic and Statistics in 1996/97, by body mass index. Overweight and obesity definitions according to bodymass index values proposed by Cole etal. which are related to adult definition.

RESULTS. It was studied a little more females in thisstudy (53.1\%). Averageage was 29 years and five months ( \pm 20 years), median was 25 years and six months. Overweight prevalence was $10.8 \%$ in children, $9.9 \%$ in adolescents and $28.3 \%$ among adults and obesity was $7.3 \%$, $1.8 \%$ and $9.7 \%$ respectively. It was noticed a gradual increase in overweight or obesity prevalence since infancy through adult age with decrease among the elderly.

CONCLUSION. Prevalence of overweight and obesity joined is higher among females. More than half of the women among 50 and 69 years old, from Northeast and Southeast regions of Brazil have overweight or obesity. There are few studies of overweight and obesity among children and adolescents and a lot of definition for obesity in children which makes more difficult the comparison of studies of prevalence among children. [Rev Assoc Med Bras 2003; 49(2): 162-6]

KEYwORDs: Obesity. Overweight. Nutritional evaluation.

\section{ReferênCIAS}

I. World Health Organization. Physical status: the use and interpretation of anthropometry. Geneva: WHO; 1995.

2. Uauy R, Albala C, Kain J. Obesity trends in Latin America: transiting from under- to overweight. J Nutr 200 I; I 3 I:893S-99S.

3. Caballero B. Symposium: obesity in developing countries: biological and ecological factors. J Nutr 200 I; I 3 I:866S-70S.
4. VischerTL,SeidellJC. The publichealth impact of obesity. Annu Rev Publ Health 200 I; 22:355-75.

5. Eckersley RM. Losing the battle of the bulge: causes and consequences of increasing obesity. Med J Aust 200 I; I 74:590-2.

6. Al-ShammariSA, Khoja T, Gad A. Communitybased study of obesity among children and adults in Ryadh, Saudi Arabia. Food Nutr Bull 200I; 22: 178-83.

7. Quetelet A. Physique sociale: ou, essai sur le développement des facultés de I'homme. Brussels, Belgium: C. Muquardt; 1869.

8. RozowskiJ, Arteaga A. The problem of obesity and its shocking characteristics in Chile. Rev Med Chile 1997; I 25: 12 17-24.

9. Belizzi MC, Dietz WH. Workshop on childhood obesity: summary of the discussion. Am J Clin Nutr 1999; 70:S I 73-5.

10. Willett WC, Dietz WH, Colditz GA. Guidelines for health weight. N Engl J Med. 1999; 34I:427-34.

II. Stevens J. Impact of age on associations between weight and mortality. Nutr Rev 2000; 58: 129-37.

12. Must A, Dallal GE, Dietz WH. Reference data for obesity: 85 th and 95 th percentiles of body mass index $\left(\mathrm{wt} / \mathrm{ht}^{2}\right)$ and triceps skinfold thickness. Am J Clin Nutr I 99 I ; 53:839-46.

13. MustA, Dallal GE, DietzWH. Reference data for obesity: 85 th and 95 th percentiles of body mass index $\left(\mathrm{wt} / \mathrm{ht}^{2}\right)$ and triceps skinfold thickness - a correction. Am J Clin Nutr 1991; 54:773.

14. Cole T], Freeman JV, Preece MA. Body mass index reference curves for the UK, 1990. Arch Dis Child 1995; 73:25-9.

15. Lindgren G, Strandell A, Cole T, Healy M, Tanner J. Swedish population reference standards for height, weight and body mass index attained at 6 to 16 years (girls) or 19 yeras (boys). Acta Paediatr 1995; 84: 10 19-28.

16. Sichieri R, Allam VLC. Avaliação do estado nutricional de adolescentes brasileiros através do índice de massa corporal. J Pediatr 1996; 72:80-4.

17. Luciano A, Bressan F, Zoppi G. Body mass index reference curves for children aged 3-19 years from Verona, Italy. Eur J Clin Nutr I 997; 5I:6-10.

18. Rosner B, Prineas R, Loggie J, Daniels SR. Percentiles for body mass index in U.S. children 5 to I 7 years of age. JPediatr 1998; I32:2 I I-2I.

19. Anjos LA, Veiga GV, Castro IRR. Distribuição dos valores do índice de massa corporal da população brasileira até 25 anos. Rev Panam Salud Publica/Am J Public Health 1998; $36: 3$.

20. Cole TJ, Bellizzi MC, Flegal KM, Dietz WH. Establishing a standard definition for child overweight and obesity worldwide: international survey. BMJ 2000; 320: I-6.

21 . Ministério do Planejamento, Orçamento e Gestão. pesquisa sobre padrões de vida 19961997. Rio de Janeiro: IBGE; 1999.

22. Dean AG, Dean JA, Coloumbier D, Burton AH, Brendei KA, Smith DC. Epi Info, version 6.04: a word processing, database, and statistics program for public health on microcomputers. Genebra: Centers for Disease Control and Prevention/World Health Organization; 1996.

23. Roberts SB, Dallal GE. The new childhood growth charts. Nutr Rev 200 ; 59:3I-6.

24. GuillaumeM.Definingobesityinchildhood:current practice. AmJ Clin Nutr 1999; 7: I26S-30S.

25. Livingstone MB. Childhood obesity in Europe: a growing concern. Public Health Nutr 200 I; 4:109-16.
26. Abrantes MM, Lamounier JA, Colosimo EA. Índice de Massa Corporal para identificar obesidade na infância e adolescência: indicações e controvérsias. Rev Méd Minas Gerais 2002; I2(3): 150-4.

27. Mokhtar N, ElatiJ, Chabir R, Bour A, Elkari K, Schlossman NP, Caballero B, Aguenaou H. Diet culture and obesity in northern Africa. J Nutr 200I; I3I:887S-92S

28. Balaban G, Silva GAP. Prevalência de sobrepeso e obesidade em crianças e adolescentes de uma escola da rede privada de Recife. J Pediatria 200I; 77:96-100.

29. Alves SS, Silva SS, Silva SRC, Ribeiro RS, Vertematti AS, Fisberg M. Avaliação de atividade física, estado nutricional e condição social em adolescente. Folha Méd 2000; I 1 9:26-33.

30. Chunning $C$. Fat intake and nutritional status of children in China. Am J Clin Nutr; 2000; 72: I368S-72S.

31. Abrantes MM, Lamounier JA, Colosimo EA. Recommendations for the use of body mass index for the classification of overweight and obese children and adolescents. Food Nutr Bull 2002; 23(3): 262-6.

32. Coitinho DC, Leão MM, Recine E, Sichieri R. Condições Nutricionais da população brasileira: adultos e idosos: Pesquisa Nacional sobre Saúde e Nutrição. Brasília: INAN/Instituto Nacional de Alimentação e Nutrição; | 99 |.

33. Lolio CA, Latorre MRDO. Prevalência de obesidade em localidade do Estado de São Paulo, Brasil, 1987. Rev Saúde Publica I 991; 23:33-6.

34. Sichieri R, Coitinho DC, Leão MM, Recine $E$, Everhart JE. High temporal, geographic, and income variation in body mass index among adults in Brazil. Am J Public Health 1994; 84:793-8.

35. Monteiro CA, Mondini L, Souza ALM, Popkin BM. Da desnutrição para a obesidade: a transição nutricional no Brasil. In: Monteiro CA. Velhos e novos males da saúde no Brasil: a evolução do país e de suas doenças. São Paulo: Editora Hucitec; 1995. p.247-55.

36. Gigante DP, Barros FC, Post CLA, Olinto MTA. Prevalência de obesidade em adultos e seus fatores de risco. Rev Saúde Publica 1997; $31: 236-46$

37. Gugelmin SA, Santos RV. Ecologia humana e antropometria nutricional de adultos Xavánte, Mato Grosso, Brasil. Cad Saúde Publica 200 I; 17:313-22.

38. Ell E, Camacho LAB, Chor D. Perfil antropométrico de funcionários de banco estatal no Estado do Rio de Janeiro/Brasil: I Índice de massa corporal e fatores sócio-demográficos. Cad Saúde Pública 1999; I5: | |3-21.

39. Anjos LA. Valores antropométricos da população adulta brasileira: resultados da Pesquisa Nacional sobre Saúde e Nutrição (tese prof. titular). Rio de Janeiro: Departamento de Nutrição Social, Universidade Federal Fluminense; 1993.

Artigo recebido: 17/12/2001 Aceito para publicação: 02/10/2002 